\title{
Effet de l'alimentation sur la croissance des pintadeaux au nord du Togo
}

\author{
Yao LOMBO ${ }^{1,3 *}$; Kokou TONA ${ }^{2,3}$, Essodina TALAKI ${ }^{2}$ et Bèdibètè BONFOH ${ }^{1}$ \\ ${ }^{I}$ Institut Togolais de Recherche Agronomique, BP 1163 Lomé, Togo. \\ ${ }^{2}$ Université de Lomé; Ecole Supérieure d'Agronomie, BP 1515 Lomé, Togo. \\ ${ }^{3}$ Laboratoire des Techniques de Production Avicole ; Université de Lomé; Faculté des Sciences, \\ B.P. 1515 Lomé, Togo. \\ *Auteur correspondant ; E-mail: yaolombo@yahoo.fr
}

\section{RESUME}

L'alimentation des pintadeaux est facteur important en élevage familial. L'objectif de cette étude a été d'évaluer l'influence du déséquilibre alimentaire sur les performances zootechniques et sanitaires des pintadeaux au Togo. Des pintadeaux de race locale et des pintadeaux chair ont reçu chacun, trois rations alimentaires aux valeurs nutritives différentes. Pour chaque type de pintadeaux, trois lots ont été constitués pour l'expérimentation réalisée de mai à juin 2015. Le lot1 a reçu une ration alimentaire moins riche en énergie; le lot 2 a reçu une ration alimentaire moins riche en protéine. Le lot de contrôle a reçu une ration alimentaire aux teneurs en protéine et en énergie normales. Les résultats ont montré que la réduction de la teneur en énergie de 7,10\% a induit une augmentation du gain de poids de 25,09\% chez les pintadeaux de type local et de 24,39 à 75\% chez les pintadeaux de race Hubbard. Par contre, la réduction de la teneur en proétéine de $11,03 \%$ a entraîné une diminution du gain de poids de 5,8\% chez les pintdeaux de type local et de 50 à $68 \%$ chez les seconds. La teneur en protéine de la ration est un facteur déterminant de la croissance du pintadeau. (C) 2018 International Formulae Group. All rights reserved.

Mots clés: Types de pintadeaux, rations alimentaires, gains de poids, mortalité, Région des Savanes, Togo.

\section{Effect of feed on growth of guinea poult in northern Togo}

\begin{abstract}
The feeding of guinea poult is an important factor in family farming. The objective of this study was to evaluate the influence of the dietary imbalance on zootechnical and sanitary performance of guinea poult in Togo. Local guinea poult and Hubbard flesh guinea poult each received three diets with different nutritional values. For each type of guinea poult, three batches were set up for the experiment conducted on May to Jun 2015. Batch1 received a lower feed ration; batch2 received a lower protein intake. The control group received a feed ration with normal protein and energy levels. The results showed that the reduction in energy content of $7.10 \%$ (batch1) induced an increase in weight gain of $25.09 \%$ at week 4 in slow-growing local-type guinea poult and 24.39 to $75 \%$ of the $1^{\text {st }}$ week to $5^{\text {th }}$ week in Hubbard guinea poult. On the other hand, the reduction of the proetal content of $11.03 \%$ (batch2) resulted in a decrease in weight gain of $5.8 \%$ in slow-growing localtype guinea poult and 50 to $68 \%$ in fast-growing guinea fowl. The protein content of the diet is a determining factor in the growth of the guinea poult.
\end{abstract}

(C) 2018 International Formulae Group. All rights reserved.

Keywords: Types of guinea poult, diets, weight gain, mortality, Région des Savanes, Togo. 


\section{INTRODUCTION}

La pintade présente une plus grande valeur marchande par rapport au poulet. Il n'existe aucun interdit quant à la consommation de la chair et des œufs de pintade. Contrairement à ce que l'on observe en élevage de poulets traditionnels, l'élevage de la pintade est plus difficile (Daouda et al., 2007 ; Boko et al., 2012). Cet élevage connaît des difficultés liées au démarrage des pintadeaux (Boko et al., 2012). L'élevage des pintadeaux fait face à de nombreuses contraintes qui sont d'ordre alimentaire, zootechnique et sanitaire. La mortalité des pintadeaux se situe généralement autour de $70 \%$ et dans le pire des cas à $100 \%$ en élevage traditionnel (Dahouda et al., 2009; Halbouche, 2010 ; Boko et al., 2012). Que ce soit en aviculture moderne ou traditionnelle, l'alimentation est de loin le poste de dépense le plus important $(70-80 \%$ des coûts de production) et est considérée comme le facteur majeure parmi les obstacles qui freinent le développement de l'aviculture en Afrique (Gueye, 1998). Dans la Région des Savanes, des études ont révélé que les aliments distribués aux pintadeaux sont assez diversifiés avec 55,66\% des éleveurs qui font le mélange d'ingrédients avant de les distribuer aux pintadeaux (Batcha, 2000; Dahouda et al., 2007 ; Lombo et al., 2018). Cependant, ces rations alimentaires apportées aux pintadeaux ne sont qu'un mélange de céréales : maïs concassé - sorgho-mil - riz ou maïs-sorgho-mil. Les sources de protéines qui sont parfois apportées en complément ne sont d'autre que des termites. Dès lors, les rations apportées aux pintadeaux présentent parfois des déficits ou des excès en teneurs en protéine ou en énergie avec des répercussions sur la croissance des pintadeaux (Sanfo et al., 2008 ; Sanfo et al., 2009; Sawadogo, 2013). La complémentation marginale apportée par les éleveurs aux pintadeaux ne permet pas d'optimiser la productivité et la rentabilité des exploitations avicoles. C'est dans cette logique que cette étude a été menée avec pour objectif de déterminer l'influence des déséquilibres en teneurs protéique et énergétique de l'aliment sur la croissance des pintadeaux.

\section{MATERIEL ET METHODES \\ Conduite de l'élevage}

L'essai a été réalisé en station à l'Antenne du Centre de Recherche Agronomique de la Savane Sèche (CRASS) de Dapaong dans la Région des Savanes au Togo. Il a porté sur 180 pintadeaux de race locale à croissance lente et sur 270 pintadeaux de chair à croissance rapide de race Hubbard (Tableau 1). Ces pintadeaux ont été obtenus par incubation artificielle et répartis dans des cages grillagées mobiles de dimensions $1 \mathrm{~m} \mathrm{x}$ $2 \mathrm{~m} \times 1 \mathrm{~m}$ (hauteur $=1 \mathrm{~m}$ ) à raison de 10 pintadeaux par $\mathrm{m}^{2}$. Les cages sont installées dans un poulailler. L'expérimentation a duré 60 jours de mai à juin 2015.

Les pintadeaux ont reçu trois types de rations alimentaires aux valeurs nutritives différentes : la ration de référence, la ration test 1 et la ration test 2 . Ainsi pour chaque race, trois lots de pintadeaux ont été constitués comme l'indique le tableau 1.

Les caractéristiques alimentaires des trois types de rations alimentaires ainsi composées sont mentionnées dans le Tableau 2. L'eau et l'aliment sont servis ad libitum aux pintadeaux chaque matin entre 7 et 8 heures.

Tous les pintadeaux ont bénéficiés d'un même programme de prophylaxie indiqué dans le tableau 3.

Le chauffage a été permanent et assuré par des ampoules électriques de 60 watts suspendues à $20 \mathrm{~cm}$ au-dessus de la litière. La température sous l'ampoule variait entre 36 et $38{ }^{\circ} \mathrm{C}$. Pendant ce temps, l'ambiance du bâtiment se situait entre 30 à $35{ }^{\circ} \mathrm{C}$. Le réglage de la température dans la cage grillagée est réalisé par rapprochement ou éloignement de l'ampoule du plancher. Les pintadeaux ont été pesés dès l'éclosion et à chaque semaine. $\mathrm{La}$ consommation alimentaire a été relevée chaque semaine. Le nombre d'individus morts a été relevé pour chaque lot. 


\section{Analyses statistiques}

Le logiciel $\mathrm{R}$, version 2.15 .2 a été utilisé pour faire les tests statistiques. Les tests réalisés sont l'analyse de variance suivie du test de Turkey HSD pour les comparaisons multiples des moyennes des différents paramètres étudiés. Les valeurs sont considérées comme significativement différents lorsque $\mathrm{p}<0,05$. Les résultats sont exprimés en moyenne plus ou moins erreur standard sur la moyenne (M \pm E.S.M).

Tableau 1 : Dispositif expérimental.

\begin{tabular}{|c|c|c|c|}
\hline Désignation & $\begin{array}{c}\text { Lot de contrôle : Ration } \\
\text { Référence }\end{array}$ & $\begin{array}{c}\text { Lot } 1 \text { : } \\
\text { Ration test } 1\end{array}$ & $\begin{array}{c}\text { Lot 2: } \\
\text { Ration test } 2\end{array}$ \\
\hline $\begin{array}{l}\text { Nombre de pintadeaux } \\
\text { locaux (type à } \\
\text { croissance lente) }\end{array}$ & 20 & 20 & 20 \\
\hline $\begin{array}{l}\text { Nombre de pintadeaux } \\
\text { race hubbard (type à } \\
\text { croissance rapide) }\end{array}$ & 30 & 30 & 30 \\
\hline $\begin{array}{l}\text { Nombre de répétition } \\
\text { par lot }\end{array}$ & 3 & 3 & 3 \\
\hline $\begin{array}{l}\text { Nature de la ration du } \\
\text { lot }\end{array}$ & $\begin{array}{l}\text { Ration alimentaire aux } \\
\text { teneurs en protéine et en } \\
\text { énergie normales; les } \\
\text { niveaux d'apports en } \\
\text { énergie et en protéines } \\
\text { étaient proches de ceux mis } \\
\text { en pratique sur le terrain }\end{array}$ & $\begin{array}{l}\text { Ration alimentaire } \\
\text { moins riche en énergie } \\
\text { mais à teneur en } \\
\text { protéine équilibrée }\end{array}$ & $\begin{array}{l}\text { Ration alimentaire à } \\
\text { teneur en énergie } \\
\text { normale mais moins } \\
\text { riche en protéine }\end{array}$ \\
\hline
\end{tabular}

Tableau 2 : Formule alimentaire et taux d'incorporation (stade démarrage et croissance).

\begin{tabular}{|c|c|c|c|}
\hline \multirow[t]{2}{*}{ Matière première } & \multicolumn{3}{|c|}{$\begin{array}{c}\text { Taux d'incorporation } \\
(\%)\end{array}$} \\
\hline & $\begin{array}{l}\text { Ration de } \\
\text { référence }\end{array}$ & Ration test 1 & Ration test 2 \\
\hline Maïs & 32 & 20 & 34 \\
\hline Mil & 27 & 32 & 34 \\
\hline Farine poisson & 10 & 12 & 8 \\
\hline Son cubé & 10 & 20 & 7 \\
\hline Soja torréfié & 20 & 15 & 16 \\
\hline Coquille & 1 & 1 & 1 \\
\hline TOTAL & 100 & 100 & 100 \\
\hline EM (kcal/kg) & 2870,88 & 2666,68 & 2868,99 \\
\hline $\mathrm{PB}(\%)$ & 21,48 & 21,47 & 19,11 \\
\hline Lys (\%) & 1,05 & 1,02 & 0,89 \\
\hline AAS (\%) & 0,73 & 0,77 & 0,66 \\
\hline $\mathrm{Ca}$ & 0,81 & 0,84 & 0,73 \\
\hline $\mathrm{P}$ disponible (\%) & 0,64 & 0,75 & 0,56 \\
\hline Rapport E/PB & 133,65 & 124,20 & 150,13 \\
\hline
\end{tabular}


Tableau 3 : Programme prophylactique.

\begin{tabular}{|c|c|c|c|}
\hline Age & Mesures sanitaires & Produits & $\begin{array}{c}\text { Mode d'administration et } \\
\text { posologies }\end{array}$ \\
\hline $\begin{array}{l}1^{\text {er }} \text { jour } \\
4^{\text {e }} \text { jour }\end{array}$ & $\begin{array}{l}\text { Eau pure } \\
\text { Prévention anti-infectieuse : } \\
\text { salmonelloses -entérites- et } \\
\text { colibacilloses }\end{array}$ & Eau potable & $\begin{array}{l}\text { Ad libitum à volonté } \\
\text { Voie orale dans l'eau de boisson : } \\
1 \mathrm{~g} / \mathrm{l} \text { d'eau pendant } 4 \text { jours } \\
\text { consécutifs }\end{array}$ \\
\hline $12^{\mathrm{e}}$ jour & $\begin{array}{l}\text { Prévention contre la } \\
\text { coccidiose }\end{array}$ & anticoccidien & $\begin{array}{l}\text { Voie orale dans l'eau de boisson: } \\
3 \mathrm{~g} / 51 \text { d'eau pendant } 5 \text { jours } \\
\text { consécutifs }\end{array}$ \\
\hline $3^{\mathrm{e}}$ Semaine & $\begin{array}{l}\text { Déparasitage interne : } \\
\text { syngamose (maladie du } \\
\text { bâillement), ascaridiose, } \\
\text { capilariose, hétérakidose } \\
\text { Vaccination contre la } \\
\text { Maladie de Newcastle }\end{array}$ & $\begin{array}{l}\text { déparasitage } \\
\text { interne }\end{array}$ & $\begin{array}{l}\text { Voie orale dans l'eau de boisson: } \\
2 \mathrm{~g} / \mathrm{l} \text { d'eau pendant } 1 \text { jour } \\
\text { En injection par voie sous cutanée } \\
0,25 \mathrm{ml} / \mathrm{sujet}\end{array}$ \\
\hline $4^{\mathrm{e}}$ Semaine & Prévention anti-infectieuse & antibiotique & $\begin{array}{l}\text { Voie orale dans l'eau de boisson : } \\
1 \mathrm{~g} / \mathrm{l} \text { d'eau pendant } 4 \text { jours } \\
\text { consécutifs }\end{array}$ \\
\hline $8^{\mathrm{e}}$ Semaine & $\begin{array}{l}\text { Déparasitage interne : } \\
\text { Ascaridiose, Téniasis, } \\
\text { Capilariose, Hétérakidose }\end{array}$ & $\begin{array}{l}\text { déparasitage } \\
\text { interne }\end{array}$ & Voie orale : $1 / 4$ Comprimé ( 1 jour) \\
\hline
\end{tabular}

Source : Lombo et al. (2014).

\section{RESULTATS}

Poids vif, consommation alimentaire, gain moyen quotidien des pintadeaux

La Figure 1 présente les courbes de croissance pondérale des pintadeaux de race locale et des pintadeaux chair de race Hubbard soumis aux rations alimentaires différentes. L'évolution du poids des pintadeaux nourris avec une ration moins riche en énergie mais à teneur en protéine équilibrée (ration test 1) est supérieure à celles des pintadeaux nourris avec une ration moins riche en protéine mais à teneur énergétique équilibrée (ration test 2) et la ration de référence.

Chez les pintadeaux de race locale, la réduction de la teneur en énergie de 2870,88 à $2666,68 \mathrm{Kcal}(7,10 \%)$ mais avec une teneur en proétéine maintenue équilibrée a provoqué une augmentation du gain de poids de 25,09\% à la $4^{\text {ème }}$ semaine. Par contre, la réduction de la teneur en protéine de $11,03 \%$ a entraîné une diminution du gain de poids de $5,8 \%$ à la $4^{\text {ème }}$ semaine (Tableau 4). Excepté la $1^{\text {ère }}$ semaine, l'analyse de la variance des moyennes des gains de poids ne montre aucune différnce significative au seuil de 5\% entre les effets des différentes rations.

Chez les pintadeaux chair de race Hubbard, la réduction de la teneur en énergie de 2870,88 à 2666,68 Kcal $(7,10 \%)$ a augmenté le gain de poids de $75 \%$ à la $1^{\text {ère }}$ semaine et de $24,39 \%$ à $25,78 \%$ de la $2^{\text {ème }}$ semaine à $5^{\text {ème }}$ semaine (Tableau 3). L'analyse de variance des moyennes des GMQ a montré un effet significatif $(\mathrm{p}<0,05)$ de la nature de la ration. Par contre la réduction de la teneur en proétéine de $21,48 \%$ à $19,11 \%(11,03 \%)$ a provoqué une diminution du gain de poids de 50 à $68 \%$ de la $1^{\text {ère }}$ semaine à la $5^{\text {ème }}$ semaine. satisfaire ses besoins énergétiques d'entretien et de croissance. Incapable d'ingérer un exédent de calories pour le transformer en graisse, il accuse un retard de croissance important lorsque le taux protéique du régime est insuffisant. Et pour éviter un tel retard, il faut disposer de régimes couvrant le besoin en acides aminés notamment la Lysine et les acides aminés soufrés (A.A.S) Blum et Leclercq (1976). Il a été montré au bénin que le poids des pintadeaux nourris aux acides 
aminés acquis localement au Bénin (lysine et méthionine ) et en Belgique est passé respectivement de 26,09 $\mathrm{g}$ à 805,12 $\mathrm{g}$ et de 28,25 g à 1079,16 g à 16 semaines d'âge (Beckers et al., 2013).

Il a été relevé que l'indice de consommation a été plus élevé chez les pintadeaux locaux comparativement aux types à croissance rapide. Les différences des performances observées entre les différents types étaient fondamentales et réconfortantes car la pintade importée a fait l'objet de sélection pour la viande alors que chez le type local, il existe encore une grande variabilité (Sanfo et al., 2008). La ration test 1 a donné le
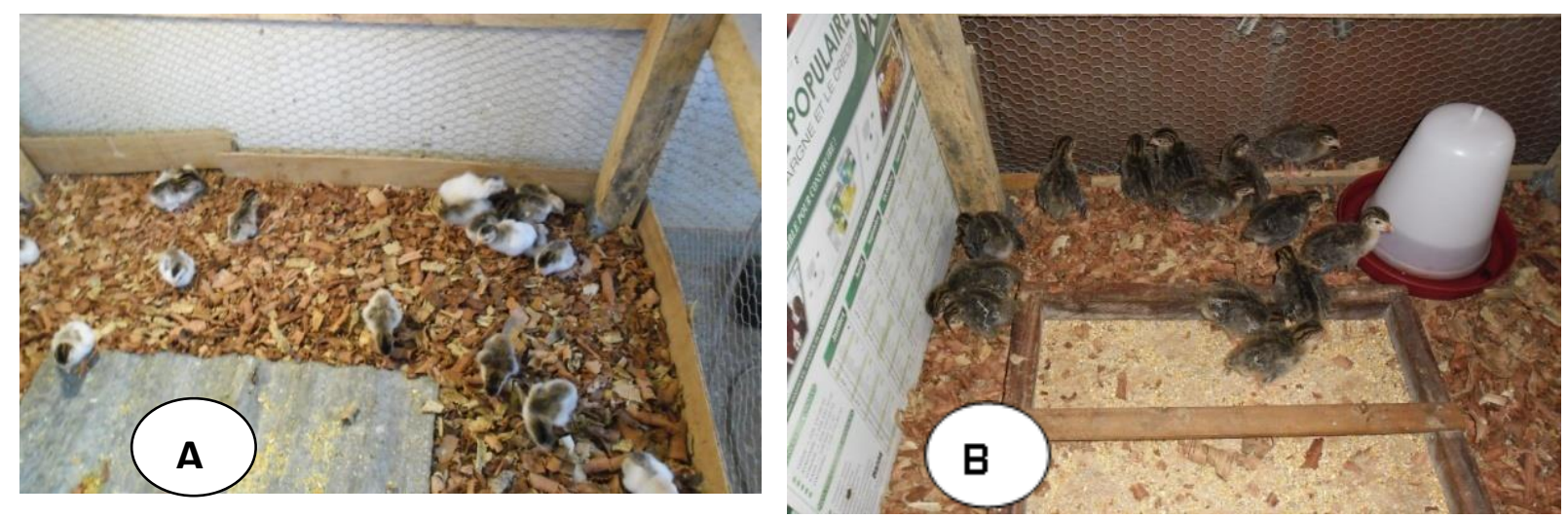

Figure 1: Démarrage des pintadeaux en cage.

A= Pintadeaux type local $; B=$ Pintadeaux type croissance rapide

Tableau 4 : Effet de la variation des teneurs d'énergie et de protéine de l'aliment sur la vitesse de croissance des pintadeaux.

\begin{tabular}{|c|c|c|c|c|c|c|c|c|}
\hline \multirow{2}{*}{ Race } & \multirow[t]{2}{*}{ Ration } & \multicolumn{7}{|c|}{ Gain moyen quotidien des pintadeaux $(\mathrm{g} / \mathrm{j})$} \\
\hline & & Semaine 1 & Semaine 2 & Semaine 3 & Semaine 4 & Semaine 5 & Semaine 6 & Semaine 7 \\
\hline \multirow{4}{*}{ 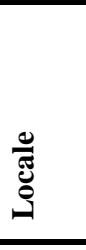 } & $\begin{array}{l}\text { Ration de } \\
\text { référence }\end{array}$ & $0,75 \pm 0,35 b$ & $2,46 \pm 0,78 \mathrm{a}$ & $1,89 \pm 1,33 \mathrm{a}$ & $2,75 \pm 2,29 a$ & $4,39 \pm 3,72 \mathrm{a}$ & $5,92 \pm 7,48 \mathrm{a}$ & $8,39 \pm 10,58 a$ \\
\hline & Ration test1 & $0,86 \pm 0,27 \mathrm{a}$ & $2,57 \pm 0,85 \mathrm{a}$ & $1,96 \pm 1,09 \mathrm{a}$ & $3,44 \pm 2,19 \mathrm{a}$ & $2,93 \pm 3,24 a$ & $9,30 \pm 6,66 \mathrm{a}$ & $4,34 \pm 9,92 \mathrm{a}$ \\
\hline & Ration test2 & $0,42 \pm 0,54 \mathrm{ab}$ & $1,58 \pm 1,048 \mathrm{a}$ & $1,35 \pm 0,73 a$ & $2,59 \pm 1,91 \mathrm{a}$ & $2,35 \pm 3,00 \mathrm{a}$ & $7,62 \pm 6,24 a$ & $1,41 \pm 7,34 \mathrm{a}$ \\
\hline & Probabilité & $0,00599 * *$ & 0,0586 & 0,439 & 0,701 & 0,466 & 0,619 & 0,345 \\
\hline \multirow{4}{*}{ 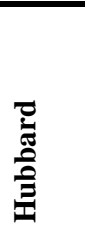 } & $\begin{array}{l}\text { Ration de } \\
\text { référence }\end{array}$ & $-0,07 \pm 0$ & $15 \mathrm{a}$ & $\begin{array}{r}2,66 \pm \\
1,58 \mathrm{ab}\end{array}$ & $2,39 \pm 2,59 b$ & $6,42 \pm 4,65 c$ & $9,77 \pm 20$ & $7 \pm 20,89 a$ \\
\hline & Ration test1 & $-0,40 \pm 0,494 a$ & $2,46 \pm 0,88 a$ & $3,04 \pm 1,68 \mathrm{a}$ & $4,11 \pm 3,39 \mathrm{a}$ & $8,65 \pm 6,22 a$ & $8,58 \pm 10,20 \mathrm{a}$ & $4,59 \pm 12,42 \mathrm{a}$ \\
\hline & Ration test2 & $-0,06 \pm 0,59 b$ & $1,76 \pm 0,82 \mathrm{a}$ & $2,14 \pm 1,51 b$ & $4,23 \pm 2,73 a$ & $3,82 \pm 4,11 b$ & $8,82 \pm 9,11 \mathrm{a}$ & $4,24 \pm 12,46 a$ \\
\hline & Probabilité & $0,000397 * * *$ & $0,0000049 * * *$ & $0,00802 * *$ & $0,000675 * * *$ & $0,000002 * * *$ & $\mathbf{0 , 8 8 2}$ & 0,872 \\
\hline
\end{tabular}

Les valeurs portant la même lettre signifient qu'il n'y a pas de différence significative.

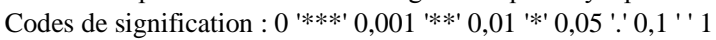




\section{DISCUSSION}

Les résultats montrent pour les deux types de pintadeaux (Figure 2) que la réduction de la teneur en protéine ou de l'énergie dans l'aliment a influencé la croissance des pintadeaux de race locale et de chair de race Hubbard.

L'étude a montré que chez les pintadeaux de race locale, la réduction de la teneur en énergie de 2870,88 à 2666,68 Kcal $(7,10 \%)$ a entraîné une augmentation du gain de poids de $25,09 \%$ à la $4^{\text {ème }}$ semaine. Par contre, la réduction de la teneur en protéine de 11, 03\% a entraîné une diminution du gain de poids de $5,8 \%$ à la $4^{\text {ème }}$ semaine (Tableau 4).

Chez les pintadeaux chair de race Hubbard, la réduction de la teneur en énergie de 2870,88 à 2666,68 Kcal $(7,10 \%)$ a augmenté le gain de poids de $75 \%$ à la $1^{\text {ère }}$ semaine et de $24,39 \%$ à $25,78 \%$ de la $2^{\text {ème }}$ semaine à $5^{\text {ème }}$ semaine (Tableau 3). Par contre la réduction de la teneur en proétéine de $21,48 \%$ à $19,11 \%(11,03 \%)$ a provoqué une diminution du gain de poids de 50 à $68 \%$ de la $1^{\text {ère }}$ semaine à la $5^{\text {ème }}$ semaine. La réduction de la teneur en énergie avec une teneur en protéine équilibrée a influencé positivement le gain de poids chez le pintadeau alors que la réduction de la teneur en protéine avec la teneur en énergie équilibrée a influencé négativement le gain de poids chez le pintadeau. Le Coz-Douin (1992) a relevé que la réduction de la teneur énergétique de 3250 à $3100 \mathrm{Kcal}(4,6 \%)$ diminuait le gain de poids de $4,1 \%$ contrairement à nos résultats. Bien que l'énergie soit réduite dans cette étude, le rapport Energie/Protéines brutes est resté dans les normes par rapport aux besoins du pintadeaux (Tableau 2). Ce qui expliquerait le gain de poids observé. Le gain de poids est lié au rapport Energie/Protéines brutes (Le CozDouin, 1992). La réduction du taux de protéine de $12,5 \%$ et $25 \%$ provoquait une diminution du gain de poids de $2,9 \%$ et $6 \%$ soit $5 \mathrm{~g}$ par point de protéine ce qui corrobore nos observations. L'effet améliorateur de la croissance des pintadeaux par la ration enrichie en protéine est égalment confirmé par Van der Horst et Clavé (2007) qui ont relevé que le poids vif des pintadeaux nourris avec un aliment démarrage pintade enrichi en protéines brutes et acides aminés (EM= 2880 $\mathrm{Kcal} / \mathrm{kg}, \mathrm{MAT}=24,5 \%$ ) était accru de manière hautement significative $(+6 \%)$ par rapport au témoin $(\mathrm{EM}=2880 \mathrm{Kcal} / \mathrm{kg}$, MAT $=23 \%$ ) (Van der Horst et Clavé, 2007). Les mêmes auteurs ont également relévé que la distribution d'aliment démarrage gibier plus riche en protéine $(\mathrm{EM}=2780 \mathrm{Kcal} / \mathrm{kg}$, MAT $=27,5 \%$ ) et d'aliment démarrage pintade enrichi en protéines brutes et acides aminés $(\mathrm{EM}=2880 \mathrm{Kcal} / \mathrm{kg}, \mathrm{MAT}=24,5 \%)$ a permis de stimuler la consommation des pintadeaux de façon significative pendant la période de distribution de 1 à 7 jours par rapport à l'aliment démarrage classique pintade (EM = $2880 \mathrm{Kcal} / \mathrm{kg}, \mathrm{MAT}=23 \%$ ). Alors que chez les deux types de pintadeaux de l'expérimentation, la consommation alimentaire a augmenté avec l'âge pour toutes les rations distribuées; Cependant, la ration test 2 , moins riche en protéines, était la ration la moins consommée comparée à la ration test 1 et la ration de référence (tableau 5). En effet, Le Coz-Douin (1992) a indiqué que le pintadeau règle sa consommation pour satisfaire ses besoins énergétiques d'entretien et de croissance. Incapable d'ingérer un exédent de calories pour le transformer en graisse, il accuse un retard de croissance important lorsque le taux protéique du régime est insuffisant. Et pour éviter un tel retard, il faut disposer de régimes couvrant le besoin en acides aminés notamment la Lysine et les acides aminés soufrés (A.A.S) Blum et Leclercq (1976). Il a été montré au bénin que le poids des pintadeaux nourris aux acides aminés acquis localement au Bénin (lysine et méthionine ) et en Belgique est passé respectivement de $26,09 \mathrm{~g}$ à $805,12 \mathrm{~g}$ et de $28,25 \mathrm{~g}$ à $1079,16 \mathrm{~g}$ à 16 semaines d'âge (Beckers et al., 2013).

L'étude a également relevé que l'indice de consommation a été plus élevé chez les pintadeaux locaux comparativement aux types à croissance rapide. Ce qui montre que les pintadeaux locaux transforment moins les aliments comparés aux pintadeaux de race hubbard. Les différences des performances observées entre les différents types étaient fondamentales et réconfortantes car la pintade importée a fait l'objet de sélection pour la viande alors que chez le type local, il existe 
encore une grande variabilité (Sanfo et al., 2008). La ration test 1 a donné le plus faible indice de consommation qui a été de 4,58 et 8,81 à la $4^{\text {ème }}$ semaine d'âge respectivement chez les pintadeaux à croissance rapide et chez ceux à croissance lente. Avec cette ration, l'indice de consommation était de 4,17 et 6,96 respectivement pour les deux types de pintadeaux à la fin de l'expérimentation. Sanfo et al. (2008) au Burkina Faso ont relevé un indice moyen de consommation de 4,7 de 0 à 8 semaines sur des pintadeaux de race locale nourris avec une formule alimentaire améliorée de démarrage dont les caractéristiques sont : protéines brutes $(\mathrm{PB})=$ $19,8 \%$; énergie brute $(\mathrm{EB})=2970 \mathrm{kcal} / \mathrm{g}$ de MS (comparable à la ration test 2).

Les mortalités ont été enregistrées dans tous les lots avec des taux faibles variant de 0 à 5,88\% chez les pintadeaux de race Hubbard et de 0 à 12,50\% chez les pintadeaux de race locale (Tableau 6). Ces taux de mortalité faibles obtenus sont comparables avec celui trouvé par Dahouda et al. (2008) soit 12\% en milieu contrôlé au Bénin. Malgré les dispositions, il faut reconnaître que l'élevage en milieu contrôlé présente des taux de mortalité plus faibles que ceux obtenus en liberté. Ceci pourrait être associé par le faible potentiel de croissance du matériel animal

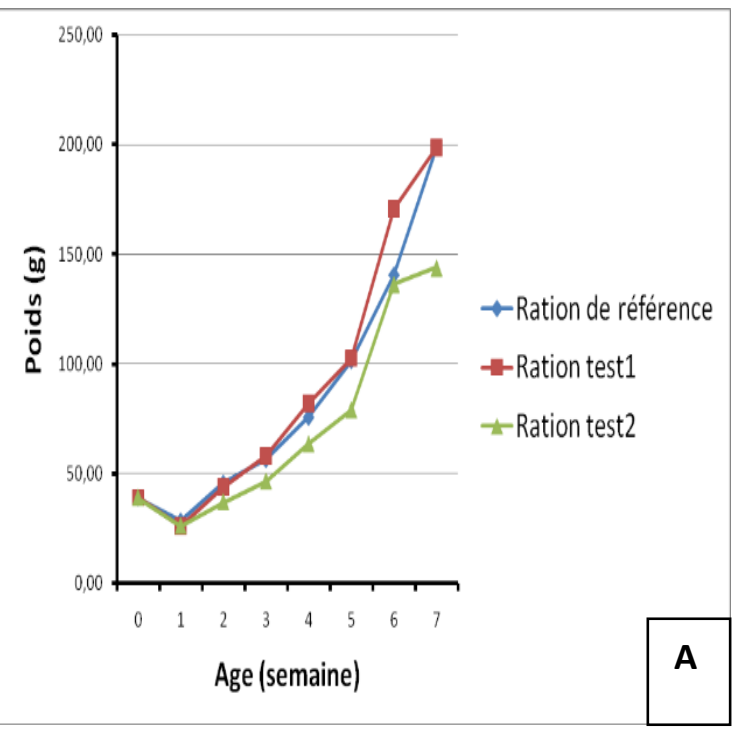

utilisé en station ou à un problème de consanguinité, ou par le stress lié à la claustration et à la température trop élevée ou encore par la présence des maladies virales dont les vaccins ne sont encore trouvés pour maîtriser toutes les pathologies de la pintade notamment l'entérite transmissible, la maladie foudroyante, la proventriculite etc. (Boko et al., 2012).

Les taux de mortalités ont été faibles avec les pintadeaux de race Hubbard comparés à ceux obtenus avec ceux de race locale. Cette différence s'expliquerait par le fait que les pintadeaux de race hubbard sont obtenus à partir des œufs des pintades provenant d'une ferme d'élevage moderne, donc beaucoup plus suivi sur le plan sanitaire alors que les pintadeaux de race locale sont issus des œufs collectés chez un éleveur traditionnel. Les pintades des élavages traditionnels sont moins suivi sur le plan sanitaire. Il a été montré que si la pintade parentale ne bénéficie pas d'un suivi sanitaire correct, les pintadeaux issus des celles-ci sont exposés à plus de pathologies (Boko et al, 2012). Le fort taux de mortalité observé chez les pintadeaux de race locale avec la ration test 1 n'est dû au régime alimentaire puis que ces pintadeaux n'ont manifesté aucun signe de fébrilité ou de baisse de performances.

Figure 2: Courbes de croissance pondérale des pintadeaux de race locale (A) et des pintadeaux chair de race Hubbard (B) soumis à trois types de rations alimentaires différentes. 
Tableau 5: Effet de la variation des teneurs d'énergie et de protéine de l'aliment sur la consommation alimentaire et l'indice de consommation.

\begin{tabular}{|c|c|c|c|c|c|c|c|c|}
\hline \multirow[b]{2}{*}{ Race } & \multirow[t]{2}{*}{ Ration } & \multicolumn{7}{|c|}{ Consommation alimentaire (g) } \\
\hline & & Semaine 1 & Semaine 2 & Semaine 3 & Semaine 4 & Semaine 5 & Semaine 6 & Semaine 7 \\
\hline \multirow{10}{*}{ } & & $10,35 \pm$ & & & $31,74 \pm$ & $44,60 \pm$ & $42,43 \pm 10,5$ & \\
\hline & Ration de & 2,77 & $14,00 \pm 1,25$ & $18,84 \pm 3,89$ & 8,57 & 9,94 & 8 & $26,23 \pm 9,88$ \\
\hline & référence & $(13,68)$ & $(5,69)$ & $(9,95)$ & $(11,54)$ & $(10,15)$ & $(7,16)$ & $(3,13)$ \\
\hline & & & & & $30,35 \pm$ & $36,09 \pm$ & $51,70 \pm$ & $30,26 \pm$ \\
\hline & Ration & $9,91 \pm 4,24$ & $14,82 \pm 0,91$ & $17,98 \pm 3,41$ & 6,98 & 10,71 & 15,43 & 17,13 \\
\hline & test1 & $(11,56)$ & $(5,81)$ & $(9,16)$ & $(8,81)$ & $(12,33)$ & $(5,56)$ & $(6,96)$ \\
\hline & & & & & $27,68 \pm$ & $37,38 \pm 12,1$ & 39,03 & $22,89 \pm$ \\
\hline & Ration & $8,75 \pm 1,72$ & $9,39 \pm 1,00$ & $15,79 \pm 4,51$ & 4,74 & 6 & $\pm 10,65$ & 21,19 \\
\hline & test2 & $(19,70)$ & $(5,66)$ & $(12,46)$ & $(10,69)$ & $(15,86)$ & $(5,12)$ & $(16,23)$ \\
\hline & Probabilité & $0,0117 *$ & $0,0176 *$ & $\mathbf{0 , 0 1 7 2} *$ & 0,0974. & NS & NS & NS \\
\hline \multirow{10}{*}{ 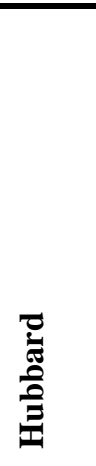 } & & & & $14,45 \pm$ & $19,71 \pm$ & & & \\
\hline & Ration de & $5,79 \pm 0,15$ & $13,44 \pm 3,76$ & 2,48 & 5,41 & $19,31 \pm 6,33$ & $18,97 \pm 4,11$ & $19,92 \pm 1,87$ \\
\hline & référence & $(-72,48)$ & $(7,19)$ & $(5,43)$ & $(8,25)$ & $(3,01)$ & $(1,94)$ & $(6,28)$ \\
\hline & & & & & & & 19,19 & $19,19 \pm$ \\
\hline & Ration & $5,94 \pm 0,00$ & $13,17 \pm 4,17$ & $14,01 \pm 2,30$ & $18,83 \pm 5,17$ & $19,02 \pm 6,21$ & $\pm 4,01$ & 0,58 \\
\hline & test1 & $(-14,51)$ & $(5,36)$ & $(4,61)$ & $(4,58)$ & $(2,20)$ & $(2,24)$ & $(4,17)$ \\
\hline & & & & 13,18 & 19,23 & 19,27 & 19,53 & $20,39 \pm$ \\
\hline & Ration & $4,66 \pm 0,59$ & $10,96 \pm 2,48$ & $\pm 2,14$ & $\pm 4,94$ & $\pm 6,27$ & $\pm 4,25$ & 0,66 \\
\hline & test2 & $(-77,69)$ & $(6,19)$ & $(6,16)$ & $(4,55)$ & $(5,05)$ & $(2,21)$ & $(4,81)$ \\
\hline & Probabilité & $0.0176 *$ & 0.0172* & NS & NS & NS & NS & NS \\
\hline
\end{tabular}

()$=$ Valeur de l'indice de consommation

Signif. codes: 0 '***' $0.001^{\text {'**' }} 0.01^{\prime} *$ ' $0.05^{\prime} .{ }^{\prime} 0.1^{\prime \prime}$ ' 1

NS : Non sigificatif

Tableau 6: Taux de mortalité des pintadeaux (\%).

\begin{tabular}{|c|c|c|c|c|c|c|c|c|}
\hline \multirow{2}{*}{ Race } & \multirow[b]{2}{*}{ Ration } & \multicolumn{7}{|c|}{ Taux de mortalité (\%) } \\
\hline & & $\begin{array}{l}\text { Semaine } \\
1\end{array}$ & $\begin{array}{l}\text { Semaine } \\
2\end{array}$ & $\begin{array}{l}\text { Semaine } \\
\mathbf{3}\end{array}$ & $\begin{array}{l}\text { Semaine } \\
4\end{array}$ & $\begin{array}{l}\text { Semaine } \\
5\end{array}$ & $\begin{array}{l}\text { Semaine } \\
6\end{array}$ & $\begin{array}{l}\text { Semaine } \\
7\end{array}$ \\
\hline \multirow{3}{*}{ 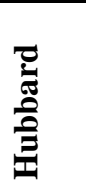 } & $\begin{array}{l}\text { Ration de } \\
\text { référence }\end{array}$ & 0,00 & 2,63 & 2,70 & 0,00 & 2,78 & 2,86 & 5,88 \\
\hline & Ration test 1 & 0,00 & 2,63 & 0,00 & 0,00 & 2,70 & 4,17 & 1,45 \\
\hline & Ration test 2 & 0,00 & 0,00 & 2,63 & 2,63 & 1,39 & 4,23 & 5,88 \\
\hline \multirow{3}{*}{ 岇 } & $\begin{array}{l}\text { Ration de } \\
\text { référence }\end{array}$ & 0,00 & 0,00 & 0,00 & 0,00 & 0,00 & 0,00 & 0,00 \\
\hline & Ration test 1 & 0,00 & 10,00 & 11,11 & 0,00 & 0,00 & 0,00 & 12,50 \\
\hline & Ration test 2 & 0,00 & 10,00 & 0,00 & 0,00 & 0,00 & 0,00 & 11,11 \\
\hline
\end{tabular}




\section{Conclusion}

L'étude a eu pour objectif de déterminer l'influence des déséquilibres en teneurs protéique et énergétique de l'aliment sur la croissance des pintadeaux. Le déséquilibre alimentaire a eu une influence sur la croissance des pintadeaux. La réduction de la teneur en énergie et la teneur en protéine maintenue équilibrée ont conduit à augmenter le gain de poids aussi bien chez les pintadeaux de race Hubbard et que ceux de race locale. Par contre la réduction de la teneur en protéine de l'aliment a entraîné une diminution du gain de poids chez les deux types de pintadeaux. La teneur en protéine dans l'aliment des pintadeaux reste un des éléments déterminants à prendre en compte dans l'élevage des pintadeaux surtout pendant les phases de démarrage et de croissance. La mise en œuvre de ces résultats auprès des éleveurs contribuera à réveiller leur conscience et à améliorer la productivité de cet élevage au Togo et dans la sous-région Ouest africaine.

\section{CONFLIT D'INTERETS}

Les auteurs déclarent n'avoir aucun conflit d'intérêts.

\section{CONTRIBUTIONS DES AUTEURS}

YL et TK ont contribué à la conception et à la planification des expérimentations. YL a conduit les expérimentations en station du Centre de Recherche Agronomique de la Savane Sèche (CRASS). YL, TE et BB ont rédigé le manuscrit. YL a réalisé les traitements statistiques. TK a supervisé les travaux. Tous les auteurs ont approuvé la version finale du manuscrit.

\section{REMERCIEMENTS}

Les auteurs remercient les étudiants en master et les thésards du Laboratoire des techniques de production avicole de l'Université de Lomé (UL) et les chercheurs et techniciens de Centre de Recherche Agronomique de la Savane Sèche (CRASS) de l'Institut Togolais de recherche Agronomique (ITRA).

\section{REFERENCES}

Batcha N. 2000. Profil pathologique des pintadeaux en élevage traditionnel dans la Région des Savanes au Togo. Mémoire. Ecole Supérieure d'Agronomie. Université de Lomé. 68 p.

Beckers Y, Bindelle J, Gengler N. 2013. Effet de la provenance et de la proportion des acides aminés (lysine et méthionine) sur les performances zootechniques de la pintade locale grise (Numidameleagris) élevée au bénin. Journal of Wuhan University of Technology Mater Sci Ed. 15 (2): 113-123.

Blum JC, Leclercq B. 1976. Besoin du pintadeau de chair en lysine et en acides aminés soufrés pendant la période de croissance et de finition. Ann. Zootech. 25: 397- 406.

Boko KC, Kpodekon TM, Dahouda M, Marlier D, Mainil JG. 2012. Contraintes techniques et sanitaires de la production traditionnelle de pintade en Afrique subsaharienne. Ann. Méd. Vét. , 156 : 2536.

Dahouda M, Senou M, Toleba SS, Boko CK, Adandedjan JC, Hornick J L. 2008. Comparaison des caractéristiques de production de la pintade locale (Numida meleagris) en station et dans le milieu villageois en zone soudano guinéenne du Bénin. Livestock Research for Rural Development, 20: 1- 12.

Dahouda M, Toleba SS, Youssao AKI, Bani Kogui S, Yacoubou A S, Hornick JL. 2007. Guinea fowl rearing constraints and flock composition under traditional management in Borgou Department, Benin. Family Poult., 17 : 3-14.

IEMVT-CIRAD. 1996. Développement de l'aviculture traditionnelle en Afrique tropicale, 1989. Fiches techniques d'élevage tropical. Maisons-Alfort, France, IEMVT-CIRAD, 8 p.

DSID, 1996.- Recensement national de l'Agriculture. Togo, $303 \mathrm{p}$.

Gnassimgbe C. N. 1983. Contribution à l'étude de l'élevage de la pintade au Togo. Thèse de Médecine Vétérinaire. Dakar. 119 p. 
Gueye EHF. 1998. Village egg and fowl meat production in Africa. Worlds Poult. Sci. J., 54 : 73-86.

Halbouche M. 2010. Performances de ponte, de reproduction et de croissance de la pintade locale Numida meleagris en Algérie ; European 1. Scientific Research, 47(3) : 320-333.

Le Coz-Douin J. 1992. L'Elevage de la Pintade. Editions du Point Vétérinaire : Maisons-Alfort, France; 252 p.

Lombo Y, Kombate DT, Dao B, Ekoue KS, Tona KJ, Gbéasso M, Gbati OB. 2014. Etude comparée de l'efficacité de trois méthodes de prévention des maladies des pintadeaux au nord du Togo. J. Rech. Sci. Univ. Lomé (Togo), Série A, 16(3): 61-67.

Lombo Y, Tona K, Bonfoh B. 2018. Analysis of the Technical and Sanitary Constraints of the Traditional Breeding of Guinea Fowl in "Région des Savanes" of Northern Togo. Journal of Pharmacy and Pharmacology, 6: 77-87.

Sanfo R, Boly H, Savadogo L, Brian O. 2008. Performances pondérales de la pintade locale (Numida meleagris) en système d'alimentation améliorée dans la zone centrale du Burkina Faso. Revue Élev. Méd. vét. Pays Trop., 61(2) : 135-140.

Sanfo R, Sanfo R, Boly H, Savadogo L, Brian O. 2009. Eléments d'analyse de l'élevage villageois de la pintade locale (Numida meleagris) dans le Plateau Central du Burkina Faso. Revue Africaine de Santé et de Productions Animales. E.I.S.M.V. de Dakar, 7 : 107 114

Savadogo B. 2013. Effet de l'incorporation du niébé dans la ration sur les performances zootechniques de la pintade locale. Mémoire de fin de cycle). Institut de Développement Rural ; Burkina Faso, 60 p.

Van der horst F, CLAVE H. 2007. Adaptation des régimes alimentaires pour une croissance optimale de la pintade sous label rouge (phase démarrage). Septièmes Journées de la Recherche Avicole, Tours, 28 et 29 mars 2007. 174177. 